\title{
Quels liens établir entre la philosophie praxialiste de David Elliott et le programme de formation de niveau primaire du Ministère de l'Education au Québec (PFEQ) dans le domaine de la musique?
}

\section{Véronique Daigle}

Volume 2, numéro 2, 2015

Une relève

URI : https://id.erudit.org/iderudit/1060136ar

DOI : https://doi.org/10.7202/1060136ar

Aller au sommaire du numéro

Éditeur(s)

OICRM

ISSN

2368-7061 (numérique)

Découvrir la revue

Citer cette note

Daigle, V. (2015). Quels liens établir entre la philosophie praxialiste de David Elliott et le programme de formation de niveau primaire du Ministère de

l'Éducation au Québec (PFEQ) dans le domaine de la musique ? Revue musicale OICRM, 2(2), 178-189. https://doi.org/10.7202/1060136ar
Résumé de l'article

En 1995, David J. Elliott publie Music Matters. A New Philosophy of Music Education. À travers cet ouvrage, l'auteur propose une nouvelle philosophie de l'éducation musicale appelée praxialisme. Cet essai vise à mettre en exergue les liens pouvant être établis entre la philosophie praxialiste d'Elliott et le programme de formation de niveau primaire du Ministère de l'éducation au Québec (PFEQ) dans le domaine de la musique. Cet essai présente les fondements et lignes directrices du praxialisme, notamment en relation avec l'élaboration de programmes de formation. Il s'attache également, afin d'obtenir un portrait éclairé de cette philosophie, aux pour et contre reliés au praxialisme. Enfin, cet essai décrit les points essentiels du PFEQ et met en lumière les différents liens qui peuvent être établis entre la philosophie praxialiste et le PFEQ. 


\title{
Quels liens établir entre la philosophie praxialiste de David Elliott et le programme de formation de niveau primaire du Ministère de l'Éducation au Québec (PFEQ) dans le domaine de la musique? \\ Véronique Daigle
}

\begin{abstract}
Résumé
En 1995, David J. Elliott publie Music Matters. A New Philosophy of Music Education. À travers cet ouvrage, l'auteur propose une nouvelle philosophie de l'éducation musicale appelée praxialisme. Cet essai vise à mettre en exergue les liens pouvant être établis entre la philosophie praxialiste d'Elliott et le programme de formation de niveau primaire du Ministère de l'éducation au Québec (PFEQ) dans le domaine de la musique. Cet essai présente les fondements et lignes directrices du praxialisme, notamment en relation avec l'élaboration de programmes de formation. Il s'attache également, afin d'obtenir un portrait éclairé de cette philosophie, aux pour et contre reliés au praxialisme. Enfin, cet essai décrit les points essentiels du PFEQ et met en lumière les différents liens qui peuvent être établis entre la philosophie praxialiste et le PFEQ.
\end{abstract}

Mots clés : éducation musicale; musique; philosophie; praxialisme; programme de formation.

\begin{abstract}
In 1995, David J. Elliott publishes Music Matters. A New Philosophy of Music Education, based on the praxial philosophy. This essay outlines a brief history of the praxial philosophy in order to better understand Elliott's motivation to develop this new philosophy of music education. The various foundations and the guidelines of the praxial philosophy are presented, particularly those that concern the elaboration of a music curriculum and the praxial philosophy's pros and cons are discussed. Finally, the essential foundations of the QEP are described in relation with Elliott's praxial philosophy.
\end{abstract}

Keywords: curriculum; music; music education; philosophy; praxialism. 
Mes expériences professionnelles en tant que pédagogue et interprète m'ont souvent amenée à réfléchir à la valeur de la musique et plus précisément à la façon adéquate de l'enseigner. Après un retour réflexif sur mes pratiques pédagogiques, l'influence d'une philosophie de l'action sur celles-ci m'est apparue évidente. Ainsi, ma réflexion s'est penchée sur le praxialisme, cette philosophie pragmatique de l'éducation musicale élaborée par David Elliott. À travers cet essai, j'ai donc voulu approfondir mes connaissances à propos de cette philosophie, mais également vérifier en quoi le programme de formation de l'école québécoise en musique (PFEQ) pouvait reposer sur des fondements plus contemporains issus de la philosophie praxialiste.

\section{ORIGINES DE LA PHILOSOPHIE PRAXIALISTE DE DAVID ElLIOTT}

Il semble intéressant, dans un premier temps, de voir ce qui a inspiré Elliott dans l'élaboration de sa philosophie praxialiste de l'éducation musicale. L'origine étymologique du praxialisme remonte à l'Antiquité grecque, ce terme provenant du mot praxis qui signifie " action ", mot déjà utilisé par Aristote au IV siècle avant Jésus-Christ (Regelski 1998). Pour Aristote, la pensée humaine est une action intelligente qui nécessite trois éléments : theoria, technè et praxis ${ }^{1}$. Plus précisément, le mot praxis utilisé par Aristote signifie la connaissance des actions adéquates à poser pour arriver à ses fins.

Bien qu'Aristote considère l'action comme étant un des éléments essentiels de la pensée humaine, la période de l'Antiquité est néanmoins caractérisée par la suprématie de l'esprit sur le corps (Garda 2003). Associé au philosophe français Descartes, ce dualisme entre la raison et les sens a alimenté les discours philosophiques durant plusieurs siècles et a grandement influencé les théories philosophiques liées au domaine musical. En effet, selon Reimer (2003), la philosophie esthétique, un domaine spécifique de la philosophie, est apparue en Europe au XVIII siècle et est caractérisée par le dualisme entre la raison et les sens. La philosophie esthétique concerne toutes les formes d'art et se divise en trois sous-théories principales : le contextualisme, le formalisme et l'expressionnisme ${ }^{2}$ (ibid.).

Malgré leur influence sur le monde musical durant plusieurs décennies, ces théories ont fait l'objet de nombreuses critiques. Essentiellement, on leur reproche

1 Selon Regelski (1998), theoria signifie la vérité absolue, technè représente les méthodes et techniques et praxis représente les moyens et actions à poser pour arriver à ses fins. Transposée au vocabulaire actuel, cette définition de la pensée humaine pourrait s'apparenter aux trois types de connaissances à la base de la psychologie cognitive, soit les connaissances déclaratives (theoria), procédurales (technè) et conditionnelles (praxis). Selon Tardif, "les connaissances déclaratives sont essentiellement des connaissances théoriques. Il s'agit [...] de la connaissance de faits, de règles, de lois, de principes. Les connaissances procédurales correspondent au comment de l'action, aux étapes pour réaliser une action; il s'agit de séquences d'actions. Les connaissances conditionnelles concernent le quand et le pourquoi des actions " (Tardif 1993, p. 4).

2 Selon Reimer (2003), le contextualisme (ou référentialisme dans les éditions de 1970 et 1989) sous-tend que la signification et la valeur d'une œuvre se trouvent à l'extérieur de celle-ci. La signification politique et sociale de l'œuvre est donc priorisée. Quant au formalisme, les qualités esthétiques d'une œuvre n'ont rien en commun avec ce qui se trouve à l'extérieur de l'œuvre. Au contraire, sa signification réside uniquement dans sa forme, soit les sons. En ce qui a trait à l'expressionnisme, la signification de l'art réside dans les qualités esthétiques et expressives de l'œuvre. 
leur vision trop étroite, abstraite et intellectuelle de la musique (Westerlund 2003) et le fait qu'elles reposent sur une série de dogmes et de mentalités peu évolutives (Reimer 2003). Ainsi, plusieurs philosophes de l'époque ne sont pas en accord avec ces idéologies (ibid.). À titre d'exemple, Dewey, qui aurait amorcé l'élaboration de sa théorie de la connaissance vers 1890, s'inspire du mouvement philosophique pragmatique lancé par Charles S. Pierce en 1879 pour contester le fameux dualisme corps/esprit (Westbrook 1993). Selon Westbrook (ibid.), Dewey accorde une grande importance à l'expérience humaine, c'est-à-dire à la nécessité d'avoir vécu personnellement quelque chose pour la connaître. Sur le plan éducatif, la vision de Dewey se traduit donc par la mise en place d'un environnement d'apprentissage qui permet à l'enfant d'expérimenter activement les savoirs en se confrontant à des problèmes qui l'amènent à faire appel à ses connaissances et à ses savoir-faire.

En dépit de ces critiques, la philosophie esthétique continue à grandement influencer l'éducation musicale entre 1930 et 1990 (Elliott 1995). Selon Elliott (ibid.), la philosophie esthétique, popularisée par Leonhard et House à partir de 1950 et par Reimer vers 1980, privilégie la perception et la contemplation des choses au détriment de l'action. Ainsi, Elliott, bien qu'il ait lui-aussi étudié et enseigné la philosophie esthétique de l'éducation musicale, est mécontent de cette pensée conventionnelle qui ne s'arrime pas à ses convictions. C'est pourquoi il élabore en 1995 une nouvelle philosophie de l'éducation musicale qu'il nomme le praxialisme ${ }^{3}$.

\section{FondEMENTS ET LIGNES DIRECTRICES DE LA PHILOSOPHIE PRAXIALISTE DE DAVID ELLIOTT}

Je me suis questionnée sur les fondements de cette philosophie, c'est-à-dire sur ce qui est à la base de cette pensée praxialiste. Le premier point important renvoie au fait qu'Elliott (1995) adhère à la théorie matérialiste qui ne fait pas de distinction entre le corps et la conscience. Elliott prône en effet une approche fondée sur le concept de l'embodiement, que l'on peut traduire par la corporéité, c'est-à-dire une approche réunissant la conscience et le corps, en considérant qu'ils ne peuvent pas être séparés l'un de l'autre (Elliott et Silverman 2012).

Deuxièmement, la philosophie praxialiste implique que le fait de faire de la musique inclut à la fois le produit et le processus. Selon Elliott (1995), le processus est lié à l'action de faire de la musique et le produit correspond au résultat du processus, soit la musique. Pour justifier son point de vue, Elliott mentionne que « la compréhension totale de la nature et de la signification de la musique comporte plus qu'une simple compréhension des œuvres musicales. La musique, entièrement comprise et enseignée, implique le processus et le produit interreliés ${ }^{4}$ » (Elliott 2005, p. 14).

3 C'est néanmoins Philip Alperson qui est le premier à utiliser le terme praxis dans le domaine de l'éducation musicale avec la parution de l'article What Should One Expect from a Philosophy of Music Education en 1991.

4 "A full understanding of the nature and significance of music involves more than an understanding of pieces or works of music. Music, fully understood and fully taught, involves processes and products interwined "; notre traduction. 
Autrement dit, le praxialisme accorde une importance au processus, sans toutefois négliger le produit.

Troisièmement, Elliott (2005) soulève l'importance d'enseigner et d'apprendre la musique comme étant une forme particulière de l'action humaine. Plus précisément, le praxialisme repose sur ces deux prémisses précises : " la nature de l'éducation musicale dépend de la nature de la musique [et] la signification de l'éducation musicale dépend de la signification de la musique dans la vie humaine ${ }^{5}$ » (Elliott 1995, p. 12). Ainsi, la musique serait essentiellement une action humaine intentionnelle, comme l'explique Elliott : la musique est " un phénomène humain multidimensionnel qui englobe deux formes entremêlées d'activités humaines intentionnelles : faire de la musique et écouter de la musique ${ }^{\prime}$ (ibid., p. 42). L'aspect multidimensionnel fait référence ici à quatre dimensions distinctes : le musicien, la musique, le fait de faire de la musique, et le contexte ${ }^{7}$. De plus, selon Elliott (ibid.), faire de la musique comprend diverses formes d'actions musicales, soit interpréter, improviser, composer, arranger et diriger. Elliott considère également qu'écouter de la musique correspond à une forme d'activité humaine en soi puisque lorsque l'humain en écoute, il donne une signification à la musique par sa façon de l'analyser. Ainsi, faire de la musique et écouter de la musique sont deux actions humaines intentionnelles et conscientes.

Quatrièmement, le praxialisme considère que l'éducation musicale doit favoriser la croissance personnelle, la connaissance de soi ainsi que le plaisir musical ${ }^{8}$. En fait, Elliott (2005) mentionne que ces valeurs devraient être accessibles, réalisables et applicables à tout apprenant et qu'elles devraient être au centre de chaque activité musicale. Pour y arriver, Elliott propose le terme musicianship que l'on pourrait traduire par la compétence musicale. Selon Elliott, le musicianship se définit comme suit : " la compétence musicale, qui inclut toujours la compétence d'écoute, est une riche forme de connaissance procédurale qui s'appuie sur quatre autres formes de savoirs musicaux ${ }^{9} »($ ibid., p. 11). En d'autres termes, la compétence musicale musicianship - est une connaissance procédurale qui comprend des connaissances formelles, informelles, impressionnistes et métacognitives ${ }^{10}$. De plus, Elliott (ibid.)

5 " the nature of music education depends on the nature of music [...] the significance of music education depends on the significance of music in human life »; notre traduction.

6 "[...] a multidimensional human phenomenon involving two interlocking forms of intentional human activity: music making and music listening "; notre traduction.

$7 \quad$ Selon Elliott (1995), le musicien est celui qui fait la musique, la musique est le produit, le fait de faire de la musique est le processus, et le contexte représente les idées et circonstances qui entourent, forment et influence ce qui est fait.

8 Notre traduction respectivemet des termes self-growth, self-knowledge et musical enjoyment (ou flow) qu'emploie Elliott (2005).

9 "Musicianship, which always includes listenership, is a rich form of procedural knowledge that draws upon four other kinds of musical knowing in surrounding and supporting ways »; notre traduction.

10 Selon Jaccard (2007), les connaissances formelles correspondent aux connaissances déclaratives; les connaissances informelles correspondent à tout ce qui s'acquiert par l'expérience musicale, mais qui est difficile à verbaliser, les connaissances impressionnistes correspondent à une sorte d'intuition que l'on peut appeler émotion cognitive, les connaissances métacognitives correspondent aux habiletés de régulation, d'autoévaluation. 
considère que tout élève peut apprendre à faire et à écouter de la musique. En fait, Elliott résume graphiquement le musicianship ainsi :

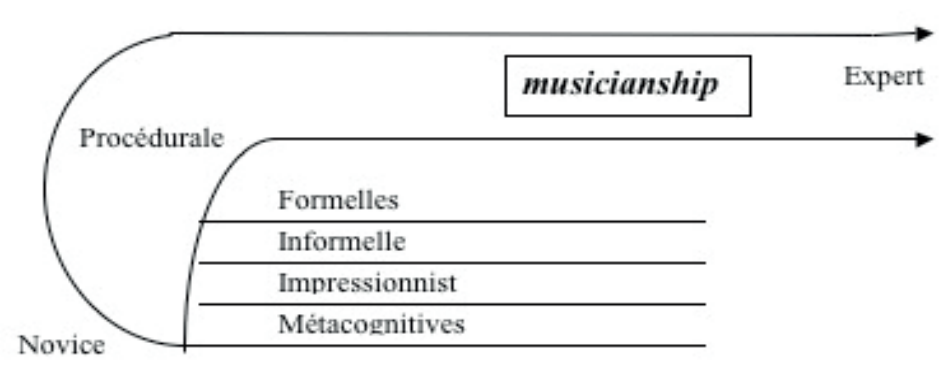

Figure 1 : Musicianship (Elliott 1995, p. 54).

En terminant, les approches pédagogiques privilégiées par le praxialisme s'apparentent grandement au courant d'apprentissage constructiviste; en effet, selon Elliott (2005), le développement des compétences musicales se réalise par l'entremise d'un processus d'apprentissage où l'élève est actif et fait preuve d'autonomie ${ }^{11}$. Ainsi, même si cela n'est pas explicitement précisé dans les propos d'Elliott, il est possible d'établir des liens entre l'approche pédagogique qu'il propose et la théorie constructiviste.

ÉLABORATION DES PROGRAMMES DE FORMATION EN MUSIQUE SELON LA PHILOSOPHIE PRAXIALISTE

Dans cette section del'essai, je propose de m'attarderàl'élaboration des programmes de formation en musique selon Elliott. Plus précisément, j'y explique comment Elliott propose d'appliquer sa philosophie à travers les programmes de formation. Tout d'abord, l'élaboration des programmes de formation en musique doit être basée sur ce qu'Elliott nomme curriculum-as-practicum, c'est-à-dire les programmes comme practicum, où les situations d'apprentissage visent à expérimenter concrètement la musique. Aussi, ces programmes comme practicum sont accessibles à tous puisque selon Elliott (1995), le concept d'habileté innée n'existe pas. Afin d'élaborer de tels programmes, Elliott propose la figure suivante comme point de départ :

11 Pour appuyer ses propos, Elliott cite Gardner : « les élèves apprennent plus efficacement lorsqu'ils sont engagés dans des projets riches et significatifs [...] lorsque les élèves ont l'opportunité de réfléchir sur leurs progrès "; notre traduction de cet extrait : "students learn effectively when they are engaged by rich and meaningful projects [...] when students have ample opportunity to reflect on their progress " (Gardner [1990] cité dans Elliott 2005, p. 12). 


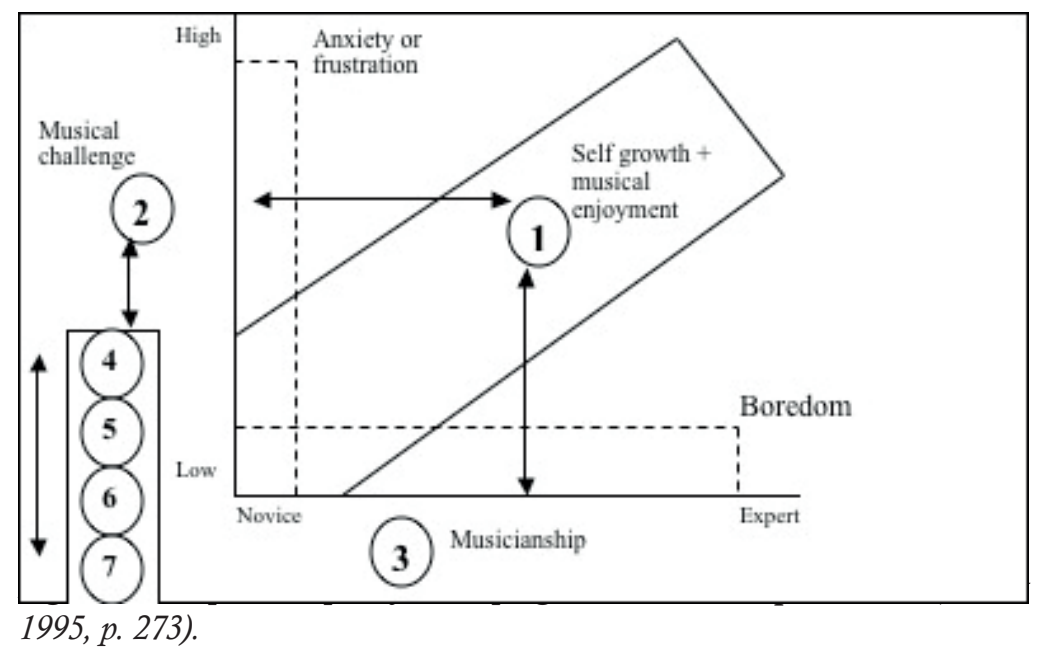

Cette figure rappelle qu'un programme d'éducation musicale doit avoir pour objectif premier le développement de la croissance personnelle, de la connaissance de soi et du plaisir musical. Ces besoins sont comblés par la mise en place de pratiques musicales visant le développement du musicianship en relation avec les défis musicaux adaptés au niveau de l'élève. Pour soutenir la motivation de l'apprenant, il est nécessaire d'atteindre un équilibre entre la difficulté de la tâche à réaliser et le niveau de compétence de l'élève. Voici plus en détail les sept points importants à intégrer à un programme de formation, selon Elliott (1995) $)^{12}$, développé dans une optique de practicum :

12 Voici de brèves définitions des stratégies d'enseignement selon Elliott (1995, p. 278-280). Modélisation : l'élève observe, écoute et construit ses apprentissages. L'enseignant le questionne par la suite. Guide : l'enseignant offre des indices, pistes et modèles pour diriger l'attention de l'élève. Support : l'enseignant offre des suggestions et soutient l'élève. Articulation: l'enseignant aide l'élève à s'exprimer par des mots afin de trouver une solution au problème à résoudre. Réflexion critique : inviter l'élève à réfléchir, à se questionner pour trouver ses forces et faiblesses. Exploration: aider l'élève à explorer les différentes possibilités pour prendre une décision et trouver une solution. Quant aux différentes étapes de l'évaluation, voici quelques précisions d'Elliott : description : collecte de données à l'aide de portfolio, vidéo, enregistrements; interprétation : analyse des données pour identifier les forces et faiblesses de l'élève; évaluation : poser un jugement et émettre des recommandations selon le résultat (ibid., p. 290). 


\begin{tabular}{|l|l|}
\hline But de l'éducation musicale & $\begin{array}{l}\text { - Interpréter, improviser, composer, arranger, diriger. } \\
\text { - L'écoute doit être travaillée en lien avec une des } \\
\text { compétences ci-dessus, et ce lors de chaque cours. }\end{array}$ \\
\hline Défis musicaux & $\begin{array}{l}\text { - Variété de musique. } \\
\text { - Exercices individuels et en groupe. }\end{array}$ \\
\hline Compétence musicale & $\begin{array}{l}\text { - L'enseignant connâ̂t le niveau de compétence de l'élève } \\
\text { afin de fixer des défis réalisables. }\end{array}$ \\
\hline Objectifs d'apprentissage & $\begin{array}{l}\text { - Objectifs à court et long terme. } \\
\text { - Objectifs spécifiques. }\end{array}$ \\
\hline Stratégies d'enseignement & \begin{tabular}{l}
-Modeler, guider, supporter, articuler, réfléchir, explorer. \\
\hline Séquence d'apprentissage
\end{tabular} \\
\hline Évaluation & $\begin{array}{l}\text { - Enseignement interactif et différencié. } \\
\text { - Enseignant spécialiste en musique. }\end{array}$ \\
\hline & $\begin{array}{l}\text { - Aide à l'apprentissage. } \\
\text { - Formative et sommative. } \\
\text { - Porte sur la compétence. } \\
\text { - Se réalise en trois étapes : description, interprétation et } \\
\text { évaluation. }\end{array}$ \\
\hline
\end{tabular}

LES POUR ET LES CONTRE DE LA PHILOSOPHIE PRAXIALISTE

Dans cette section, je me suis intéressée aux points de vue de différents auteurs sur les aspects positifs et négatifs de la philosophie praxialiste.

Les pour

Selon Regelski (2005), la philosophie praxialiste est centrée sur 1'humain et est accessible à tous. L'utilisation d'un large répertoire musical ainsi que plusieurs façons différentes de faire de la musique ${ }^{13}$ font en sorte que cette philosophie n'est pas réservée qu'à un petit nombre de connaisseurs comme le souligne l'auteur : "La musique n'est pas seulement pour les experts ou pour une élite de quelques connaisseurs; c'est aussi pour les conditions terre à terre de la vie quotidienne ${ }^{14}$ "(ibid., p. 235). En accord avec les propos de Regelski, Gruhn mentionne que le praxialisme est une philosophie sociale et ouverte sur le monde : «En aucun temps dans notre histoire professionnelle nous avons eu une vision de l'éducation musicale aussi centrée sur l'humain et axée sur l'action que celle présentée dans Music Matters ${ }^{15} »$ (Gruhan 2005, p. 98).

De plus, selon Regelski (2005) et Elliott (1995), le praxialisme est une philosophie qui implique l'élève dans son apprentissage, ce qui, du coup, le motive : «Le programme

13 Il existe différentes façons de faire de la musique selon la philosophie praxialiste : improviser, composer, arranger, diriger, interpréter.

14 "Music is not only for experts or an elite few connoisseurs; it is also of and for the down-to-earth conditions of everyday life »; notre traduction.

15 "At no other time in our professional history did we have a view of music education that is as people-focused and as action-oriented as that presented in Music Matters »; notre traduction. 
comme particum est hautement motivant pour les élèves et les enseignants ${ }^{16}$ » (Elliott 1995, p. 270). En effet, Regelski (2005) mentionne que lorsque l'apprenant est amené à faire de la musique de façon active, il est alors plus motivé.

Dans le même ordre d'idée, Bowman explique que le praxialisme permet un apprentissage concret et efficace. En effet, cette philosophie prône l'utilisation de connaissances procédurales et conditionnelles qui rendent l'élève actif et favorisent ainsi un apprentissage concret et efficace comme le mentionne Bowman : "Le praxialisme offre des conseils pratiques judicieux pour l'instruction musicale; les compétences sont plus efficacement apprises lorsqu'il y a immersion dans la pratique elle-même ${ }^{17}$ » (Bowman 2005, p. 70).

Les contre

Selon Reimer (2003), Elliott mentionne que le processus, c'est-à-dire la façon de créer la musique, est plus important que le produit, soit le résultat musical. Le praxialisme d'Elliott présente le processus comme le seul moyen d'apprendre la musique. En d'autres termes, selon Reimer, la seule et unique façon de développer la compétence musicale serait de «faire " de la musique. Ainsi, tout enseignement qui négligerait cet aspect échouerait pour éduquer musicalement des individus. Or, selon Reimer (1996), le fait de mettre l'accent uniquement sur la performance ne suffit pas pour éduquer de façon complète des individus sur le plan musical. En accord avec Reimer, Bowman (2005) mentionne que ce n'est pas parce que la musique nécessite une action humaine qu'elle doit être axée essentiellement sur la pratique musicale.

Selon Cutietta et Stauffer (2005), le praxialisme affirme que celui qui fait de la musique, soit le musiqueur, deviendra un auditeur plus compétent que celui qui ne fait pas de musique. Autrement dit, Elliott met l'accent sur le fait d'être un musiqueur pour s'avérer un bon écouteur. Encore une fois, les compétences d'interprète, soit la pratique musicale, sont donc trop mises à l'avant-plan.

Dans le même ordre d'idées, Määttänen mentionne que le praxialisme d'Elliott ne suffit pas à lui seul pour cultiver la musicalité. Bien sûr, jouer d'un instrument de musique promeut sans aucun doute les compétences musicales et les capacités d'écoute, mais l'auteur précise que ce n'est pas l'unique façon de cultiver la musicalité comme le montre la citation suivante : "Jouer d'un instrument de musique promeut sans aucun doute l'habileté à écouter de façon significative, mais ça ne peut être l'unique façon de cultiver la musicalité, comme il semble être le cas dans le praxialisme d'Elliott $^{18}$ » (Määttänen 2003, p. 68). L'auteur ne fournit cependant pas de détails supplémentaires sur les autres moyens de cultiver la musicalité.

En terminant, comme le praxialisme d'Elliott met l'action de faire de la musique au centre de l'éducation musicale, l'écoute musicale est alors placée au deuxième rang

16 "The music curriculum-as-practicum tends to be highly motivating for students and teachers "; notre traduction.

17 "Praxialism offers sound practical guidance for musical instruction; skillful practices are most effectively learned by immersion in the practices themselves "; notre traduction.

18 "Playing musical instruments undoubtedly promotes one's ability to listen meaningfully, but it cannot be the only way to cultivate musicality, as seems to be the case in Elliott's praxialism. At least this aspect is overemphasized "; notre traduction.

Revue musicale OICRM, volume 2, n² 
(Koopman 2005). Selon Koopman, Elliott considère l'auditeur comme étant inférieur au musicien, car il négligerait l'écoute musicale dans sa posture philosophique : "Plusieurs enseignants en musique considèrent que faire et écouter de la musique sont des activités d'importance égale. Cependant, Elliott favorise fortement le fait de faire de la musique, particulièrement jouer d'un instrument de musique ${ }^{19}$ " (ibid., p. 86). En accord avec ces propos, Reimer (2003) mentionne que ceux qui adhèrent au praxialisme voient l'écoute musicale comme une activité passive, inférieure et moins créative que le fait de faire de la musique.

\section{CONCLUSION}

En ce qui me concerne, je me positionne en faveur du praxialisme, car cette philosophie de l'éducation musicale offre de nombreux avantages (efficace, concret, accessible à tous, ouvert sur le monde, motivant...) qui permettent d'obtenir de bons résultats si elle est utilisée adéquatement dans l'enseignement. De plus, je considère que la plupart des critiques formulées à l'égard du praxialisme sont fondées sur une mauvaise interprétation et/ou sur une certaine exagération des propos d'Elliott ${ }^{20}$. La philosophie praxialiste d'Elliott a une grande influence sur mes pratiques pédagogiques. Que ce soit en contexte d'enseignement scolaire ou extrascolaire, je mets en application plusieurs éléments tirés du praxialisme; j'accorde beaucoup d'importance à rendre l'élève actif lors des situations d'apprentissage, je vise le développement de compétences chez l'élève afin que celui-ci comprenne la pertinence des savoirs, je tente d'inclure toutes les façons possibles de faire la musique en utilisant une variété de styles musicaux, je m'efforce d'offrir une pédagogie différenciée adaptée aux besoins de l'élève... Ainsi, comme la philosophie praxialiste répond à mes besoins en tant qu'enseignante, je l'utilise quotidiennement afin de guider mes pratiques pédagogiques.

\section{Liens entre la philosophie praxialiste et le PFEQ}

Suite à la présentation de la philosophie praxialiste de David Elliott, la section suivante met en évidence les liens pouvant être établis entre le praxialisme et le programme de formation de niveau primaire du Ministère de l'Éducation au Québec (PFEQ) dans le domaine de la musique.

Le praxialisme accorde une importance à la réalisation de programmes d'enseignement axés sur le développement de compétences (interpréter, composer, diriger, écouter,

19 "Many music educators treat musicing and listening as equally important activities. However, Elliott strongly favors musicing, particularly performing »; notre traduction.

20 Prenons par exemple Reimer (2003) qui mentionne que le praxialisme voit l'écoute musicale comme une activité passive, inférieure et moins créative que le fait de faire de la musique. Cette critique m'apparaît démesurée, car Elliott insiste sur le fait qu'écouter de la musique est une activité musicale au même titre que de jouer de la musique. De plus, dans son ouvrage Music Matters. A New Philosophy of Music Education (1995), Elliott consacre un chapitre entier à l'écoute musicale où il mentionne qu'elle doit faire partie de toute activité musicale. 
improviser et arranger). En plus de favoriser les différentes façons de faire de la musique, ce fondement du praxialisme met également l'accent sur le développement de connaissances procédurales chez l'élève. Or, ces éléments de la philosophie praxialiste se retrouvent dans l'approche pédagogique prônée par le PFEQ comme le montre la citation suivante : « le programme de formation de l'école québécoise se caractérise essentiellement par le choix de développer des compétences ${ }^{21}$ et par l'attention portée à la démarche d'apprentissage » (Ministère de l'Éducation du Québec 2001, p. 4). Les compétences disciplinaires et les connaissances procédurales sont donc au cœur de ce programme. Quant aux compétences poursuivies par ce dernier, elles sont les mêmes que celles proposées par Elliott puisque l'on y retrouve les compétences inventer (qui comprend improviser, arranger et composer), apprécier et interpréter. En ce qui a trait à l'organisation de ces compétences dans les situations d'apprentissage, Elliott propose l'omniprésence de l'écoute musicale en lien avec l'interprétation ou la création ${ }^{22}$. Or, c'est tout à fait ce que propose le PFEQ puisque chaque situation d'apprentissage en musique devrait permettre à l'élève d'activer au moins deux des trois compétences disciplinaires, soit inventer ou interpréter et apprécier (ibid., p. 239). Donc, même si les termes diffèrent, la compétence apprécier, ou l'écoute musicale selon Elliott, doit être travaillée auprès des enfants, et ce, lors de chaque cours.

L'autre élément important de l'approche d'Elliott réside dans la qualité de la formation des enseignants en musique; ceux-ci doivent détenir une spécialisation en musique. Il en va de même pour le MELs qui propose, à travers le PFEQ, que chaque enseignant de musique obtienne préalablement sa formation de pédagogue en musique.

L'éducation multiculturelle d'Elliott implique la présence de divers styles musicaux provenant de diverses cultures du monde. Dans le PFEQ, il est mentionné que l'enseignant doit offrir une formation ouverte sur le monde, où l'élève est appelé à inventer, interpréter et apprécier des pièces musicales de multiples provenances. Donc, autant dans ce programme que dans la vision praxialiste, on retrouve un souci d'enseigner et de faire découvrir un répertoire varié à l'élève.

Quant aux approches pédagogiques préconisées dans les situations d'enseignement, Elliott propose plusieurs stratégies inspirées du constructivisme que l'enseignant peut utiliser : modélisation, guide, support, articulation, réflexion critique et exploration. Il est donc important que l'élève et l'enseignant interagissent ensemble et que l'enseignant guide l'élève dans l'action (Elliott 1995). On reconnaît ici des éléments du courant constructiviste qui sont également présents dans le PFEQ : " beaucoup d'éléments du programme de formation font appel à des pratiques basées sur une conception de l'apprentissage d'inspiration constructiviste. Dans cette perspective, l'apprentissage est considéré comme un processus dont l'élève est le premier artisan »

21 «Compétence : savoir-agir fondé sur la mobilisation et l'utilisation efficaces d'un ensemble de ressources " (Ministère de l'Éducation du Québec 2001, p. 4).

22 Selon Elliot, l'élève doit développer sa compétence musicale et sa capacité d'écoute à travers ces activités : " performing-and-listening, improvising-and-listening, composing-and-listening, arranging-and-listening, conducting-and-listening » (Elliott 2005, p. 7). 
(Ministère de l'Éducation du Québec 2001, p. 5). Il y est ajouté que « l'enseignant est un médiateur entre l'élève et les savoirs et il est de son devoir de créer un environnement éducatif incitant l'élève à jouer un rôle actif dans sa formation " (ibid., p. 6). En somme, le PFEQ et la philosophie praxialiste proposent tous les deux un environnement d'apprentissage où l'élève est actif et où l'enseignant guide l'apprentissage.

Quant à l'évaluation, Elliott propose de nombreuses stratégies ${ }^{23}$ qui font en sorte que l'évaluation est une composante importante du processus d'apprentissage. De plus, Elliott précise que l'évaluation doit porter sur les compétences à développer. Or, dans le PFEQ, l'évaluation porte également sur le développement de compétences, et elle est utilisée dans une perspective d'aide à l'apprentissage : "L'évaluation fait partie intégrante de la démarche d'apprentissage. Pour être en consonance avec le programme, elle doit porter sur les compétences dont il propose le développement » (ibid.). De plus, tout comme Elliott, ce programme propose l'utilisation du portfolio comme outil d'évaluation.

En terminant, l'éducation humaniste d'Elliott vise un but particulier, soit la croissance personnelle, la connaissance de soi et le plaisir musical. Il s'agit ici d'une vision plus globale de l'apprentissage de l'élève, car on considère ce dernier comme un citoyen en devenir. Cette approche philosophique se retrouve également dans le PFEQ puisqu'à travers les trois missions de la réforme scolaire (instruire, qualifier, socialiser), les activités d'apprentissage privilégient le développement de compétences générales essentielles, tant pour le parcours scolaire que pour la vie sociale de l'enfant. De plus, la présence des compétences transversales ${ }^{24}$ montre un souci de développement global de l'élève afin de favoriser sa croissance personnelle.

À la lumière de cette réflexion, nous pouvons conclure qu'il existe des liens inéquivoques entre la philosophie praxialiste d'Elliott et le PFEQ.

\section{BiBLIOGRAPHIE}

Bowman, Wayne D. (2005), "The Limits and Grounds of Musical Praxialism », dans Elliott 2005, p. 52-78.

Cutietta, Robert A., et Sandra L. Stauffer (2005), « Listening Reconsidered », dans Elliott 2005, p. 123141.

Elliott, David J., et Marissa Silverman (2012), «Rethinking Philosophy, Re-Viewing Musical-Emotional Experiences », dans Wayne D. Bowman et Ana L. Frega (dir.), The Oxford Handbook of Philosophy in Music Education, New York, Oxford University Press, p. 37-62.

23 Voici les différentes stratégies d'évaluation proposées par Elliott (1995) : l'évaluation formative, l'auto-évaluation, la métacognition, le portfolio, la collecte de données à l'aide de projets, $\mathrm{CD}$, et vidéos.

24 « [...] les compétences transversales correspondent à des savoir-agir fondés sur la mobilisation et l'utilisation efficaces d'un ensemble de ressources. Elles ont toutefois ceci de particulier qu'elles dépassent les frontières des savoirs disciplinaires tout en accentuant leur consolidation et leur réinvestissement dans les situation concrètes de la vie, précisément en raison de leur caractère transversal » (Ministère de l'Éducation du Québec 2001, p. 12). On y retrouve les compétences intellectuelles, méthodologiques, communicationnelles ainsi que personnelles et sociales. 
Elliott, David J. (2005), Praxial Music Education. Reflections and Dialogues, New York, Oxford University Press.

Elliott, David J. (1995), Music Matters. A New Philosophy of Music Education, New York, Oxford University Press.

Garda, Michela (2003), "Esthétique. Petite histoire des conceptions du beau musical », dans Jean-Jacques Nattiez (dir.), Musiques. Une encyclopédie pour le XXI siècle, "Vol. 2. Les savoirs musicaux ", Arles/Paris, Actes Sud/Cité de la musique, p. 649-671.

Gruhn, Wilfried (2005), «Understanding Musical Understanding », dans Elliott 2005, p. 98-111.

Jaccard, Sylvain (2007), «Quelle pourrait être l'évolution de la conception philosophique de l'écoute musicale selon le paradigme praxialiste, si David J. Elliott prenait en compte les critiques formulées à son égard? ", Recherche en éducation musicale, vol. 25, p. 31-62.

Koopman, Constantijn (2005), «The Nature of Music and Musical Works », dans Elliott 2005, p. 7997.

Määttänen, Pentti (2003), «Aesthetic Experience and Music Education », Philosophy of Music Education Review, vol. 22, no 1, p. 63-70.

Ministère de l'Éducation du Québec (2001), Programme de formation de l'école Québécoise, Québec, Bibliothèque et Archives nationales du Québec.

Regelski, Thomas A. (1998), "The Aristotelian Bases of Praxis for Music and Music Education as Praxis ", Philosophy of Music Education Review, vol. 6, n 1, p. 22-59.

Regelski, Thomas A. (2005), "Curriculum. Implications of Aesthetic Versus Praxial Philosophies", dans Elliott 2005, p. 219-248.

Reimer, Bennett (1996), «David Elliot's "New" Philosophy of Music Education. Music for Performers Only ", Bulletin of the Council for Research in Music Education, vol. 128, p. 59-89.

Reimer, Bennett (2003), A Philosophy of Music Education. Advancing the Vision, Upper Saddle River, Prentice Hall.

Tardif, Jacques (1993), "L'évaluation dans le paradigme constructiviste », dans René Hivon (dir.), L'évaluation des apprentissages. Réflexions, nouvelles tendances et formation, Sherbrooke, Éditions du CRP, p. 27-56.

Westbrook, Robert B. (1993), «John Dewey », Perspectives. Revue trimestrielle d'éducation comparé, vol. 23, $\mathrm{n}^{\circ} 1$, p. $277-293$.

Westerlund, Heidi (2003), " Reconsidering Aesthetic Experience in Praxial Music Education », Philosophy of Music Education Review, vol. 11, n 1, p. 45-62. 